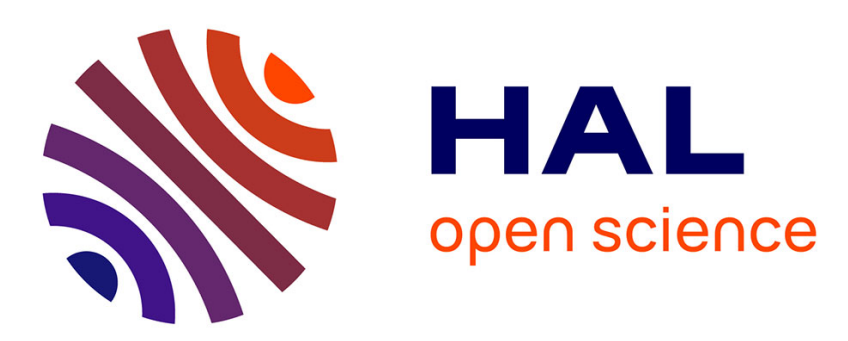

\title{
Modeling of the multiscale dispersion of nanoparticles in a hematite coating
}

\author{
Enguerrand Couka, François Willot, Dominique Jeulin, Mona Ben Achour, \\ Anthony Chesnaud, Alain Thorel
}

\section{- To cite this version:}

Enguerrand Couka, François Willot, Dominique Jeulin, Mona Ben Achour, Anthony Chesnaud, et al.. Modeling of the multiscale dispersion of nanoparticles in a hematite coating. Journal of Nanoscience and Nanotechnology, 2015, 15 (5), pp. 3515-3521(7). 10.1166/jnn.2015.9855 • hal-00965908

\section{HAL Id: hal-00965908 \\ https://hal.science/hal-00965908}

Submitted on 25 Mar 2014

HAL is a multi-disciplinary open access archive for the deposit and dissemination of scientific research documents, whether they are published or not. The documents may come from teaching and research institutions in France or abroad, or from public or private research centers.
L'archive ouverte pluridisciplinaire HAL, est destinée au dépôt et à la diffusion de documents scientifiques de niveau recherche, publiés ou non, émanant des établissements d'enseignement et de recherche français ou étrangers, des laboratoires publics ou privés. 


\title{
Modeling of the multiscale dispersion of nanoparticles in a hematite coating
}

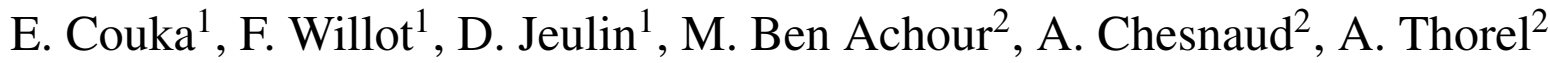 \\ ${ }^{1}$ Center for Mathematical Morphology, Mines ParisTech, 77300 Fontainebleau, France \\ ${ }^{2}$ Centre for Materials, Mines ParisTech, 10 rue Henri-Auguste Desbruères, 91003 Evry, France.
}

\begin{abstract}
Images of a hematite-based epoxy coating are obtained by scanning electron microscopy (SEM). At the scale of a few micrometers, they show aggregates of hematite nano-particles organized along thin curved channels. We first segment the images and analyze them using mathematical morphology. The heterogeneous dispersion of particles is quantified using the correlation function and the granulometry of the embedding (epoxy) phase. Second, a two-scales, 3D random microstructure model with exclusion zones is proposed to simulate the spatial distribution of particles. This simple model is parametrized by four geometrical parameters related to the exclusion zones solely. The microstructure is numerically optimized, in the space of morphological parameters, on the granulometry of the embedding epoxy phase and on the microstructure correlation function, by standard gradient-descent methods. Excellent agreement is found between the SEM images and our optimized model. Finally, the size of the representative volume element associated to the optimized microstructure model is compared with that of the SEM images.
\end{abstract}

Keywords: Microstructure optimization; Random media; Nanomaterials; Granulometry; Coating

\section{INTRODUCTION}

Hematite nanoparticles of various aspherical shapes have been synthesized and characterized for use in optical materials $[1,2]$ or for magnetic properties [3], ranging from pseudo-cubic [4] to cigar-like hematite particles [5]. At a higher length scale, the dispersion of hematite particles is most often heterogeneous [6]. However, the multiscale distribution of nanoparticles, embedded in a resin, has not been studied to the same extent. Some microstructure models have been proposed in other contexts. Jean et al. [7] devised a multiscale Boolean models to represent the dispersion of carbon black in rubber. Azzimonti et al. [8] introduced deposit models to simulate the dispersion of nanoparticles in optical materials. Such models are important to predict the behavior of nanomaterials. The multiscale dispersion strongly influences their effective (i.e. macroscopic) properties. For linear conducting materials, numerical computations have shown that the presence of aggregates of highly-conducting inclusions increase the overall properties as compared to more homogeneous dispersions [9]. In nanomaterials, the typical length scales of the particles's spatial distribution is often much larger than the inclusions themselves but of the same order as wavelength of light. Accordingly, taking dispersion into account is especially important for predicting the optical properties of composites [10].

This work is devoted to the numerical modeling of the spatial distribution of nanoparticles of hematite in a epoxy matrix. We present the material and segment greylevel SEM microstructure images in Sec. (2). In Sec. (3) we extract the correlation function and granulometry of the nanoparticles and of the epoxy. In Sec. (4) we introduce a simple two-scales microstructure model to represent the microstructure. The parameters of our model are optimized according to correlation function and granulometry criteria, as defined in Sec. (3). Results are presented in Sec. (5). We conclude in Sec. (6).

\section{Hematite nanocubes coating}

\subsection{Dispersion of nanoparticles}

In this work, we consider $\mathrm{Fe}_{2} \mathrm{O}_{3}$ iron oxide (hematite) nanoparticles embedded in a epoxy resin. The hematite particles, shown at high resolution in Fig. (1), were specifically synthesized to resemble cubes [11].

The hematite nanoparticles were blended in an epoxy resin with a concentration of $10 \%$ in mass. The nanoparticles and resin were first mixed to- 
gether in a mortar until the resulting paint becomes homogeneous. Using a bar coater technology, the mixture was then spread onto a thick and flexible shrink-wrap support in order to obtain a thin coating. For that purpose, an Elcometer 4340 motorised film applicator was used. This versatile, rugged and precise manufacturing process ensures a smooth, reproducible and consistent application of coatings, such as paint, varnish, cosmetics, glue and ceramics, without any ridge associated with such a technique. The bar coater is indeed equipped with a highly-engineered aluminium table, much smoother than a glass support, and heating element, if suited. It was accurately calibrated using a coordinate measuring machine to reach a high level of flatness. The average variation of coating thickness on the Elcometer table is about $\pm 2.3 \mu \mathrm{m}$ while it is equal to $\pm 12.0 \mu \mathrm{m}$ on glass of some low-cost tables. During the deposition, the roller translates towards the coating direction but does not spin round. The deposition speed may be set up from 0.5 to $10 \mathrm{~cm}$ per second and coating as thin as $5 \mu \mathrm{m}$ may be fabricated. In this work, $150 \mu \mathrm{m}$ thick coatings were fabricated.

As seen in Fig. (1), the particles shape are similar to dice with rounded edges and corners.

The length of the diagonal of the cubes's faces, measured from the SEM images, is about $300 \mathrm{~nm}$. The SEM image in Fig. (1a) shows the spatial dispersion of nanoparticles at higher length scales. Hematite particles aggregate around "empty regions" almost entirely made of epoxy. Around those areas, they form curved "channels" (surfaces in 3D) and some clusters of particles in regions where the channels join. Along channels, Hematite particles are quite closely packed as shown in Fig. (1b). Clearly, the dispersion exhibits a multi-scale microstructure with at least two scales: the particles average length and the typical size of the channels.

The rest of this work is devoted to the analysis and modelling of the nanoparticles spatial arrangements. The formation of the channels and their physical interpretation, beyond the scope of this work, is not investigated.

\subsection{Image segmentation}

Hereafter, we focus on 4 randomly-selected SEM images of $950 \times 950$ pixels, representing a region

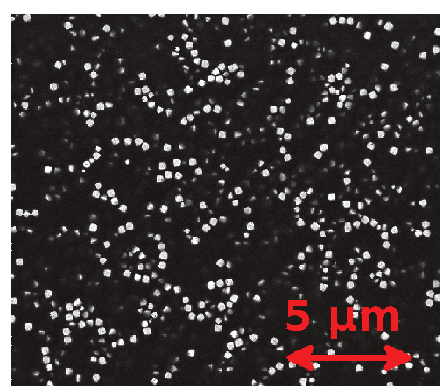

(a)

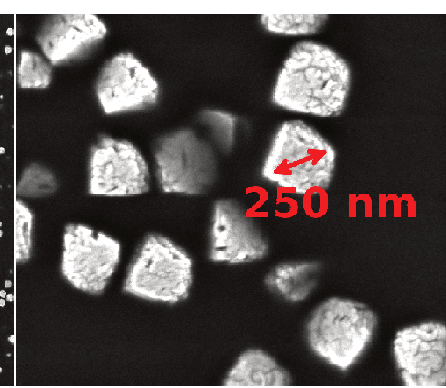

(b)
Fig. 1: Nanocubes of hematite (bright) in a epoxy resin (dark) (a); same material with scale magnified 10 times (b).

of area $30 \mu \mathrm{m} \times 30 \mu \mathrm{m}$. The images's resolution is $31 \mathrm{~nm}$ per pixel. We segment them according to a three-steps process illustrated in Fig. (2). We first apply a gaussian filter to erase noise and smoothen the background. Second, we separate nanoparticles from the resin by maximization of the interclass variance [12], an automatic segmentation method that does not need any parameter. Third, we apply an alternate sequential filter (Fig. 2). As seen in Fig. (1), SEM images show nanoparticles that are slightly beneath the plane of observation as smaller, darker particles. To eliminate these, we remove inclusions with an area smaller than 10 pixels (about $10 \times 10 \mathrm{~nm}^{2}$ ). The final result is shown in Fig. 2 (right). Fig. (3) shows two out of the four segmented images. We measure a surface fraction close to $f_{0}=9.4 \pm 0.5 \%$ on the four images.

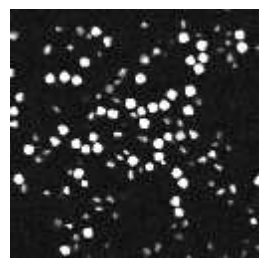

(a)

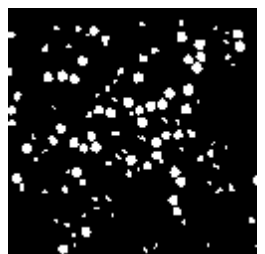

(b)

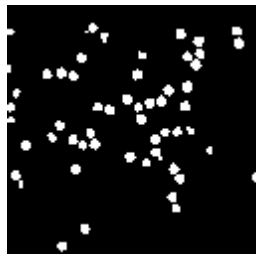

(c)
Fig. 2: Segmentation of SEM images (enlargement): original grayscale image (a), noise-filtering and thresholding (b), removing of small inclusions (c).

\section{Morphological measurements}

In this section, we use correlation functions and granulometry distributions to quantify the dispersion of nanoparticles in the resin. 

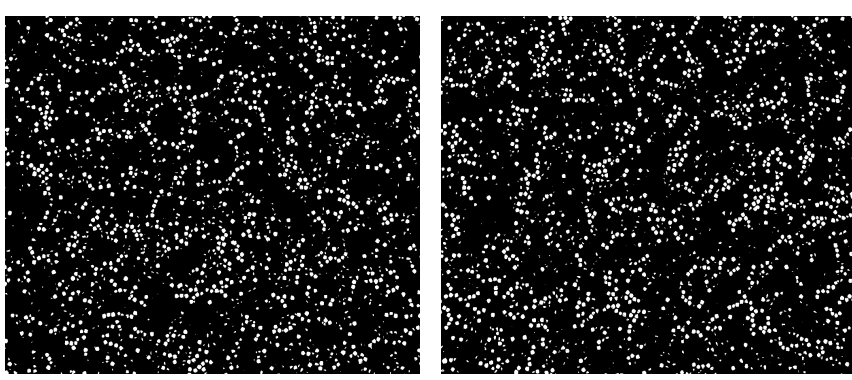

Fig. 3: Two of the four segmented SEM images, shown at the same scale.

\subsection{Correlation function}

We define the covariance function as the probability:

$$
C(\mathbf{r})=P\{\mathbf{x} \in \mathcal{H}, \mathbf{x}+\mathbf{r} \in \mathcal{H}\},
$$

where $\mathcal{H}$ is the union of hematite nanoparticles in the microstructure, and $\mathbf{x}$ is a point. At large distances $|\mathbf{r}| \gg 1$ the two events $\mathbf{x} \in \mathcal{H}$ and $\mathbf{x}+\mathbf{r} \in \mathcal{H}$ become uncorrelated and $C(\mathbf{r}=\infty) \approx C(0)^{2}$. Thus, we define the normalized covariance, or correlation function, as:

$$
\bar{C}(\mathbf{r})=\frac{C(\mathbf{r})-C(0)^{2}}{C(0)[1-C(0)]} .
$$

Taking vectors $\mathbf{r}=r \mathbf{e}_{1}(r \geq 0)$ aligned with the horizontal axis $\mathbf{e}_{1}$, we observe nearly identical correlation functions for each sample, as shown in the inset of Fig. (4). We also observe little difference along the vertical and horizontal axis, shown in Fig. (4) as well, so that the microstructure is almost isotropic as far as correlation functions are concerned. Hereafter, we note $\bar{C}(r)$ the correlation function $\bar{C}(\mathbf{r})$ irrespective of the orientation of the vector $\mathbf{r}$. The average over samples and over the horizontal and vertical directions of the correlation functions is referred to hereafter as "correlation of the SEM images".

In Fig. (4), the tangent slope at the origin $r=0$, shown in brown, cuts the abscissa at $r \approx 155 \mathrm{~nm}$. This length is to be interpreted as the mean chord length of nanoparticles. It is consistent with the length of the diagonal of the nanocubes faces, of about 300 nm, measured from Fig. (1).

\subsection{Granulometry}

We define the cumulative granulometry by openings of the epoxy phase as the conditional proba-

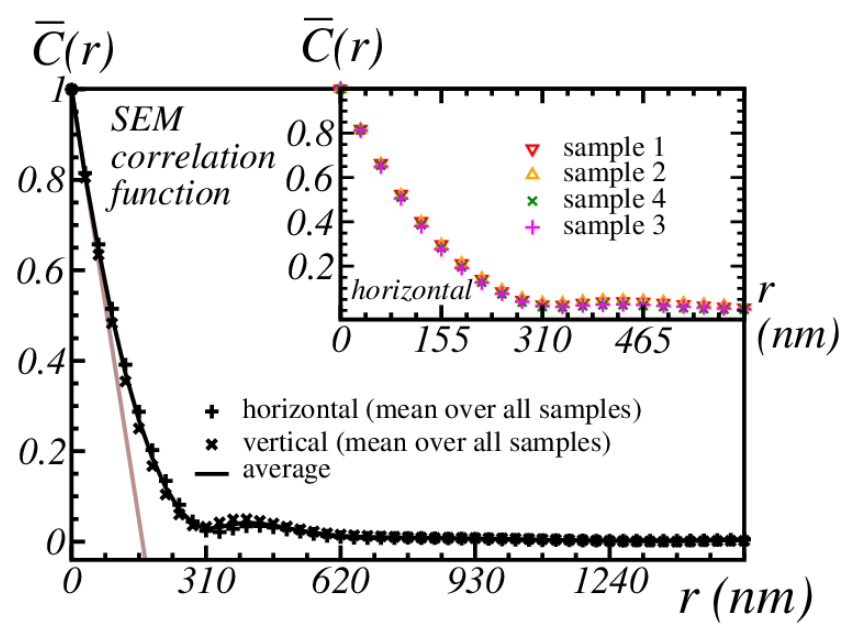

Fig. 4: Symbols: mean over all samples of the correlation functions $\bar{C}(r)$ of segmented SEM images in the horizontal and vertical directions. Black solid line: mean over the two directions (brown: tangent at $r=0$, see text). Inset: correlation function $C(r)$ for $r \leq 62 \mathrm{~nm}$ in the horizontal direction, for each sample.

bility:

$$
G(s)=\frac{P\{\mathbf{x} \in \mathcal{E}\}-P\{\mathbf{x} \in \mathcal{E}(S ; s)\}}{P\{\mathbf{x} \in \mathcal{E}\}}
$$

where $x$ is a point in the image, $\mathcal{E}$ is the epoxy phase (complementary of $\mathcal{H}$ ) and $\mathcal{E}(S ; s)$ is the morphological opening of $\mathcal{E}$ by the structural element $S$ dilated by size $s$. We use the first and second neighbors, i.e. a square of $3 \times 3$ pixels, as structural element $S$ whereas $s$ take on values that are multiples of the pixel size. The granulometry defined above is a measure of the cumulative size distribution in $\mathcal{E}$. We compute $G(s)$ independently for each of the 4 samples. The resulting curves, shown in Fig. (5) are close to each other, except at large sizes $s>700 \mathrm{~nm}$. The granulometry of sample 2 is slightly higher than the others in this domain. We hereafter neglect these variations and take the mean of all four curves, referred to as "granulometry of the SEM images" (Fig. 5).

We emphasize our use of the epoxy phase $\mathcal{E}$ rather than $\mathcal{H}$ in the granulometry. The former only is sensible to the spatial dispersion of nanoparticles. Indeed, the granulometry of the nanoparticles is zero for sizes larger than 4 voxels and contains little information (not shown). By contrast, the granulometry $G(s)$ is non null for $s \leq s_{0}$ with $s_{0} \approx 2.5 \mu \mathrm{m}$. The value $s_{0}$ is an estimate of the maximum diameter of regions without hematite. Similarly, the granulometry $G(s)$ gives 
the mean diameter of these regions, estimated to $1.05 \mu \mathrm{m}$.

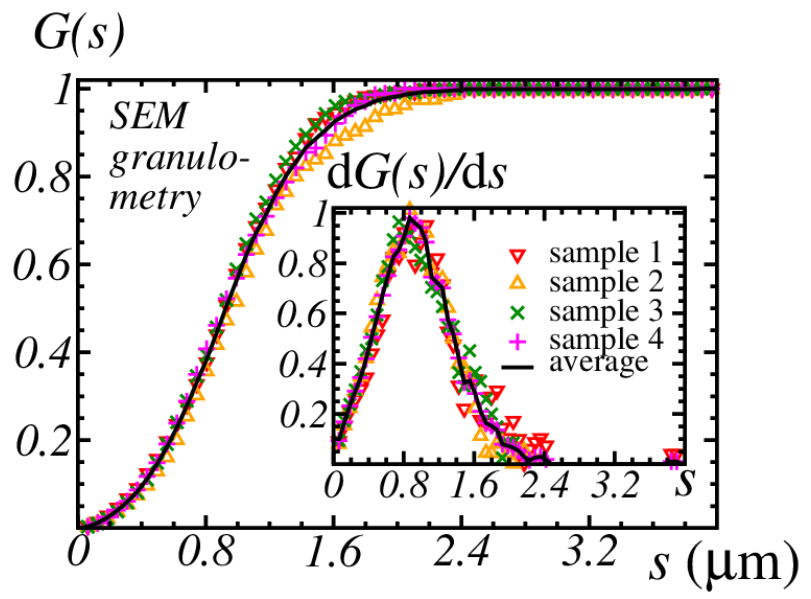

Fig. 5: Cumulative granulometry $G(s)$ of the complementary of SEM images (symbols) as a function of the size $s$ in nanometers. Solid line: average over all 4 samples. Inset: granulometry distribution function $\mathrm{d} G(s) / \mathrm{d} s$

\section{Microstructure Model}

We now introduce a random 3D microstructure model to represent the nanoparticles dispersion in the epoxy. In our model, we assume that all nanoparticles are identical (perfect) cubes with uniformly-oriented random orientations. This simplification allows us to focus on the modeling of the spatial dispersion of particles, which is our main interest. More evolved models involving size or shape distributions of particles are straightforward extensions of this work. Regions without hematite particles (or with little of them) will be hereafter referred to as "exclusion zones" in our model, which is described according to the three steps below.

First, we generate exclusion zones by a set of hard-core spheres, determined by the spheres volume fraction $f$, their diameter $a$ and a repulsion distance $\Delta$. When generating sphere centers, points closer to $\Delta$ from another point are skipped. We add spheres until the volume fraction $f$ of exclusion zones is obtained. Accordingly, a portion of exclusion zones intersect whenever $\Delta<a$.

In a second step, we place nanocubes according to a deposit model that simulates an isotropic packing of hematite particles [13]. The total

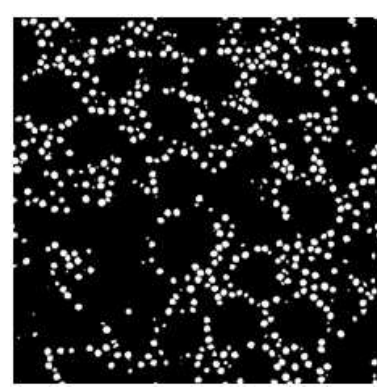

(a)

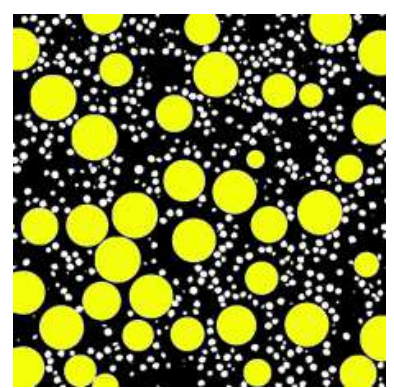

(b)
Fig. 6: 2D cut of a realization of the two-scale microstructure model (a); same image with exclusion zones shown in yellow (b).

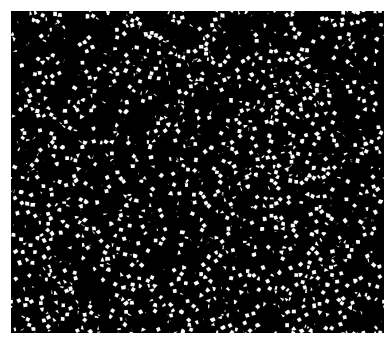

(a)

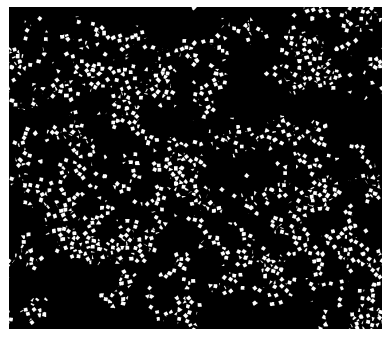

(b)
Fig. 7: Effect of the size of exclusion zones on the microstructure model: $a=310 \mathrm{~nm}(\mathrm{a}), 1860 \mathrm{~nm}(\mathrm{~b})$ with other parameters fixed to $f=10 \%, f^{\prime}=\Delta=0$.

number of nanocubes is determined as a random variable following Poisson's law. Their average number is deduced from the volume fraction of nanocubes, measured from SEM images. We start by inserting the fraction $1-f^{\prime}$ of nanocubes lying outside exclusion zones. The center of each nanocube is, initially, generated randomly in the domain, and moved in a random, isotropicallydistributed direction until a certain criterion is met. There are two possibilities. If the cube initially cuts a previously-inserted nanocube, or an exclusion zone, we move it and insert it as soon as it is entirely out of the exclusion zones and of other nanocubes. Conversely, if the cube initially does not cut a previously-inserted nanocube nor an exclusion zone, we move it until it touches either one. We insert it at the last position before the intersection occurred.

In a third step, the fraction $f^{\prime}$ of cubes lying inside exclusion zones are inserted. We use a similar packing method: if the cube center is inside an exclusion zone and the cube does not cut a previously-inserted nanocube, it is moved in a ran- 


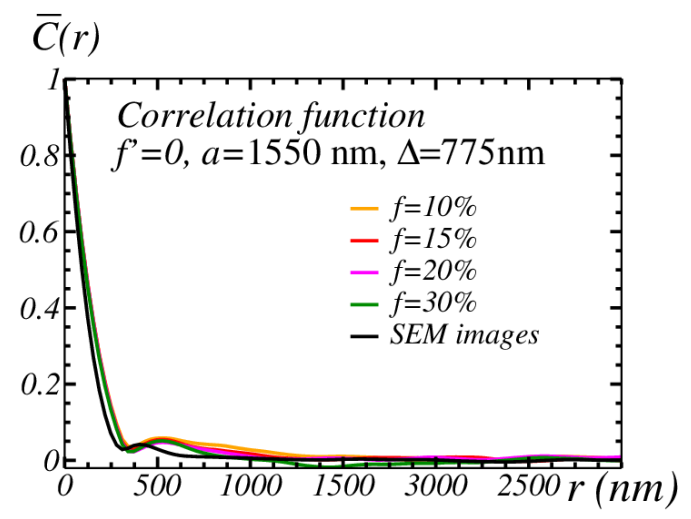

(a)

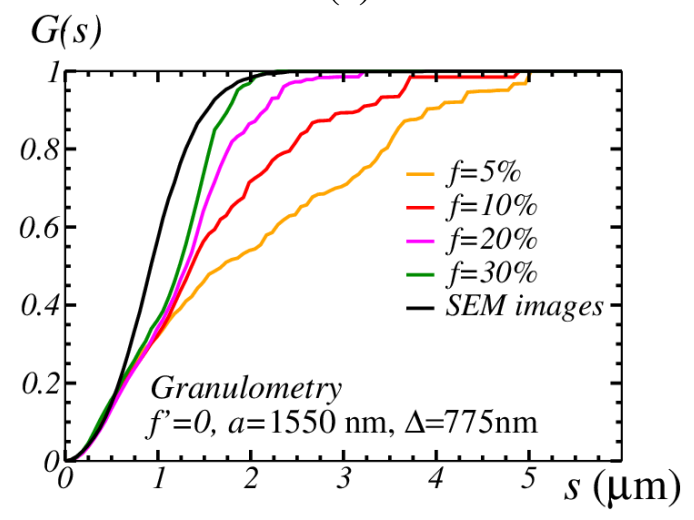

(b)

Fig. 8: Effect of the volume fraction $f$ of exclusion zones on the correlation function (a) and granulometry (b) with other parameters fixed.

dom direction until it touches a nanocube or its center is out of exclusion zones. Conversely, if the cube initially is out of an exclusion zone or intersects an already-inserted cube, we move it until its center is inside an exclusion zone and the cube does not intersect any other cube.

We emphasize that nanoparticles never intersect each other, but, instead, are "packed" together. An example of the generation of a microstructure with $\Delta=f^{\prime}=0$ is shown in Fig. (6). Exclusion zones are represented in yellow (right), the final microstructure is shown left. Examples of microstructures with $\Delta=f^{\prime}=0$, and exclusion zones volume fraction $f=10 \%$ are shown in Fig. (7) with small (left) and large (right) exclusion zones diameters $a$.

As predicted, the volume fraction $f$ of excluding zones has an important effect on the granulometry as shown in Fig. 8 (bottom) for $f^{\prime}=0$, $a=1550 \mathrm{~nm}$ and $\Delta=775 \mathrm{~nm}$. When $a$ is small, this effect is less (not shown). For the same values of the three parameters $f^{\prime}=0, a=1550 \mathrm{~nm}$

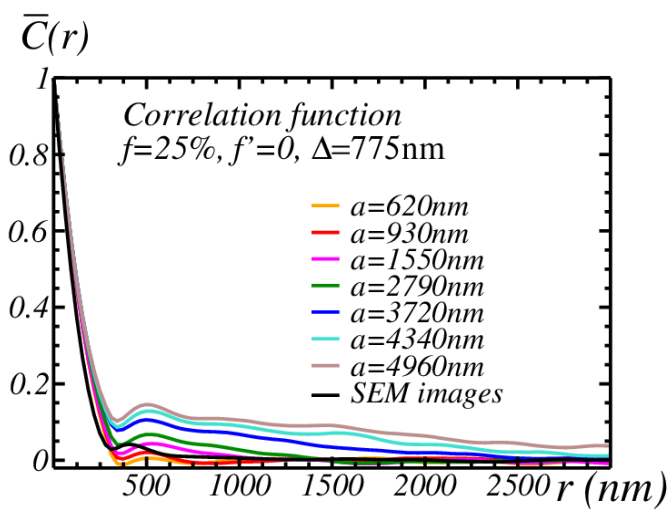

(a)

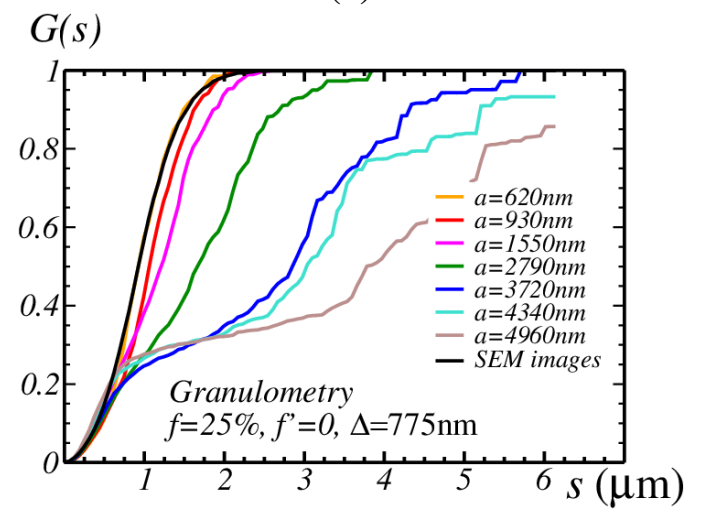

(b)

Fig. 9: Effect of the diameter $a$ of exclusion zones on the correlation function (a) and granulometry (b) with other parameters fixed.

and $\Delta=775 \mathrm{~nm}$, the effect of $f$ on the granulometry is small. A similar strong effect of the diameter $a$ is observed on the granulometry but also, as expected, on the correlation function (Fig. 9). This effect occurres whether at high or low volume fractions $f$ (not shown).

The included volume fraction $f^{\prime}$ and the repulsion distance $\Delta$ have little influence on the correlation (not shown). The former predictably influences the granulometry, whereas the repulsion distance $\Delta$ only marginally changes it (Fig. 10).

\section{Microstruture optimization AND REPRESENTATIVE VOLUME ELEMENT}

We hereafter optimize the microstructure model parameters by minimizing

$\eta_{1} \int_{h} \mathrm{~d} r\left[\overline{C^{\mathrm{M}}}(r)-\overline{C^{\mathrm{SEM}}}(r)\right]^{2}+\eta_{2} \int_{h} \mathrm{~d} s\left[G^{\mathrm{M}}(s)-G^{\mathrm{SEM}}(s)\right]^{2}$

where the correlation function $\overline{C^{\mathrm{M}}}(r)$ and granulometry $G^{\mathrm{M}}(s)$ are measured on the generated mi- 


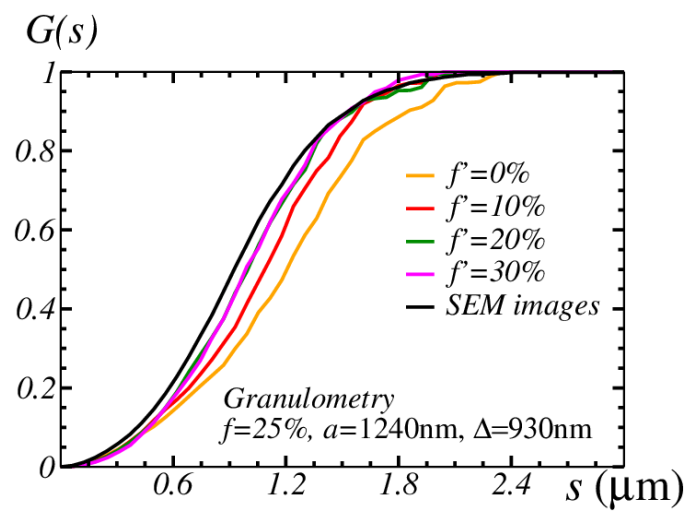

(a)

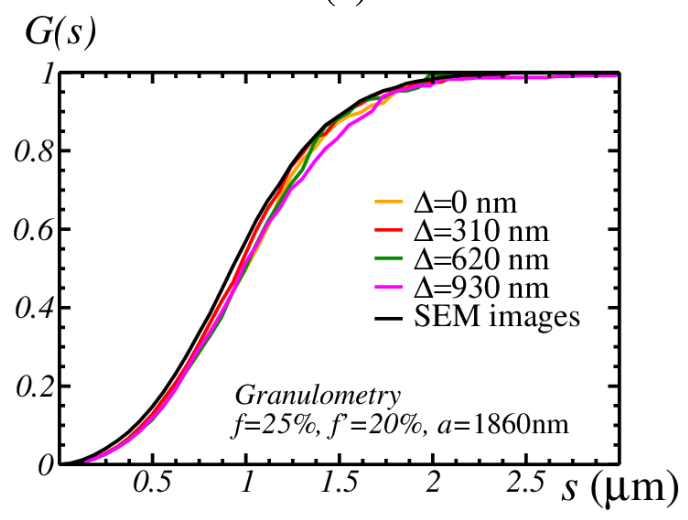

(b)

Fig. 10: Effect of the volume fraction $f^{\prime}$ of hematite in exclusion zones (a) and of the hardcore parameter $\Delta$ (b) on the granulometry, with the other 3 parameters fixed.

crostructure models whereas $\overline{C^{\mathrm{M}}}(r)$ and $G^{\mathrm{M}}(s)$ refer to the known SEM correlation functions and granulometry, resp. The criterion above allows us to optimize on both the correlation function and on the granulometry. Both quantities are comprised between 0 and 1 . The weights $\eta_{1}>0$ and $\eta_{2}>0$ are used to favor one over the other. Hereafter we make the arbitrary choice $\eta_{1}=1$, $\eta_{2}=1-f_{0} \approx 0.91$.

We use the standard gradient descent and Levenberg-Marquardt methods [14]. The latter has been successfully used for optimizing multiscale microstructure models [7]. Numerical optimization is undertaken on 3D microstructures containing $800 \times 800 \times 200$ voxels. Random 2 D cuts normal to the third axis, containing $800 \times 800$ pixels, are used to compute criterion (4). Based on the results of Sec. (3), we use $f=40 \%$, $a=2.48 \mu \mathrm{m}, f^{\prime}=10 \%$ and $\Delta=310 \mathrm{~nm}$ as initial point. Both methods converge to a local minimum. The method of Levenberg-Marquadt takes

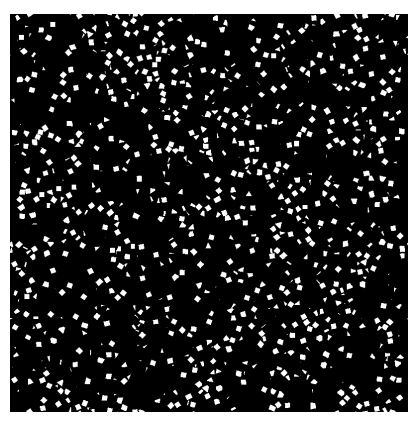

(a)

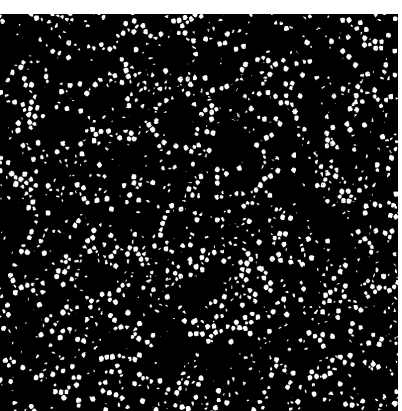

(b)
Fig. 11: Optimized numerical microstructure (a) and one segmented SEM image sample (b) observed at the same scale. Each image represents a square of size $24.8 \mu \mathrm{m}$ with resolution $31 \mathrm{~nm}$ per pixel. The parameters of the optimized model are $f=25 \%, a=$ $1862 \mathrm{~nm}, f^{\prime}=20 \%, \Delta=620 \mathrm{~nm}$.

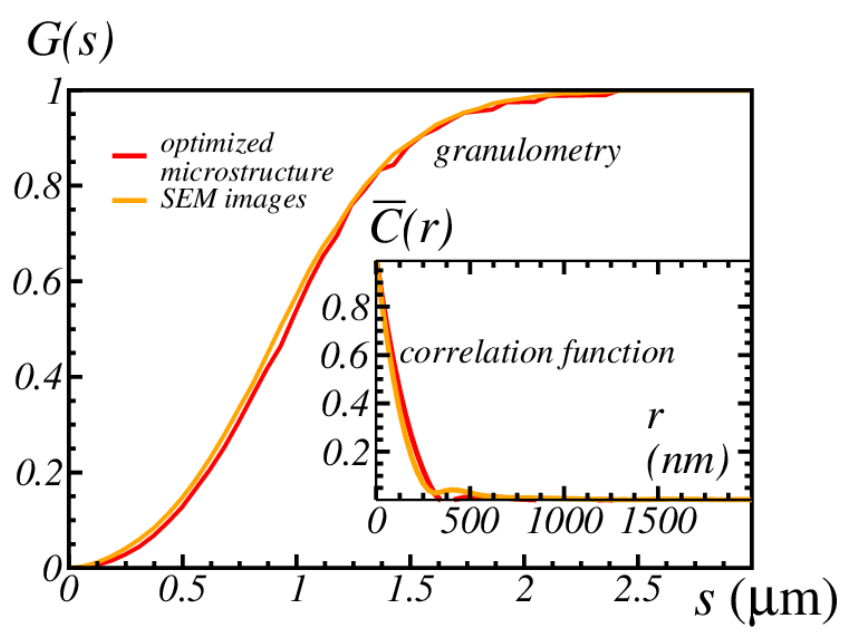

Fig. 12: Granulometry of the optimized numerical microstructure and of SEM images. Inset: correlation function.

more time at each iteration than the standard gradient descent, but uses about 4 times less iterations to converge to a given precision. We also find that both method converges to the same (local) optimum with parameters: $f=25 \%, a=1.862 \mu \mathrm{m}$, $f^{\prime}=20 \%, \Delta=0.62 \mu \mathrm{m}$. A $2 \mathrm{D}$ cut of the optimized model is represented in Fig. (11) and compared to a segmented SEM image.

The model reproduces the arrangements of nanoparticles along curved channels (necklaces) seen in SEM images.

The granulometry and correlation functions of the optimized microstructure model and of the SEM images are shown in Fig. (12). In terms of these two criteria, the optimized model is very 
close to the SEM images.

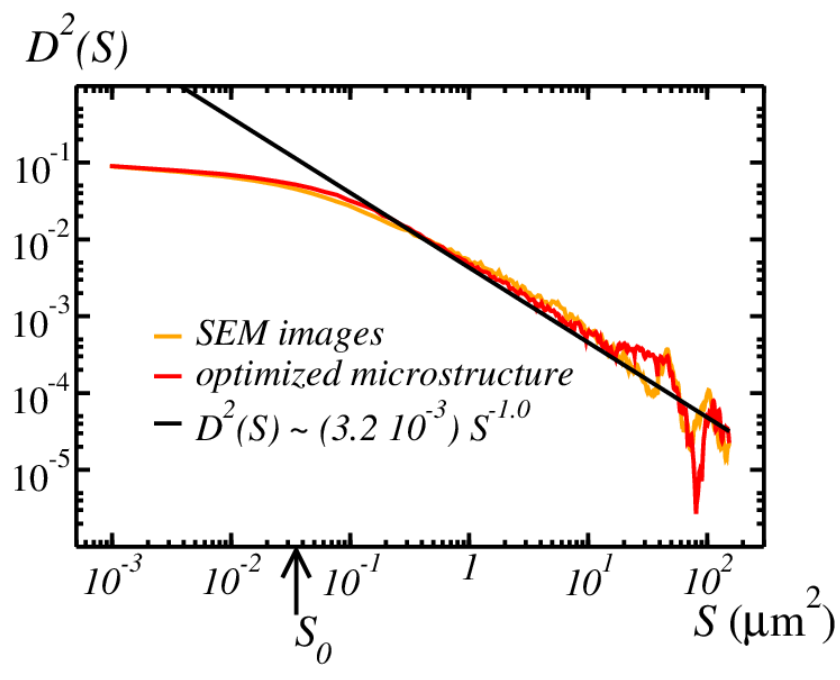

Fig. 13: Variance $D^{2}(S)$ of the apparent surface fraction of nanoparticles computed over a domain of surface area $S$ : comparison between SEM images and optimized microstructure model. Solid black line: fit of the variance $D^{2}(S)$ for $S \gg S_{0}$. Black arrow: integral range $S_{0}$.

Finally, as a complementary examination, we compute the representative volume element (RVE) related to the nanoparticules dispersion. Let $D^{2}(S)$ be the variance of the surface fraction of nanoparticles measured over independent 2D domains of area $S$ in the material. For large areas $S$, the following asymptotic behavior is recovered [15]:

$$
D^{2}(S) \sim \frac{f_{0}\left(1-f_{0}\right) S_{0}}{S}, \quad S \gg S_{0}
$$

where $S_{0}$ is the integral range and $f_{0} \approx 10 \%$ is the surface fraction of nanoparticles (Sec. 2.2). The integral range $S_{0}$ is determined by the covariance $C(\mathbf{r})$ [16]. The quantity $D(S)$ gives the confidence interval for the estimation of the surface fraction of nanoparticles on $N$ domains of area $S$ [17]. The absolute error of such estimate is:

$$
\epsilon_{\mathrm{abs}}=\frac{2 D(S)}{\sqrt{N}}
$$

To compute numerically $D^{2}(S)$, we divide one SEM image into $n \times n$ non-overlapping squares of area $S$ and compute the surface fraction of nanoparticles over each subdomain. We approximate $D^{2}(S)$ as the variance of the obtained set of surface fractions. The same computation is undertaken along a random 2D cut of our optimized microstructure model. The two curves are represented in Fig. (13). Overall, the standard deviation function of our optimized model is close to that of the SEM images, although this quantity is not (explicitly) involved in criterion (4). A region of interest $S>1.50 \mu \mathrm{m}^{2}$ is selected. Powerlaw fits on the SEM and optimized models give $D^{2}(S) \sim$ $3.1410^{-3} S^{-0.96}$ and $D^{2}(S) \sim 3.5810^{-3} S^{-0.97}$, respectively, consistently with Eq. (5). The former is represented in Fig. 13 (solid black line). From this fit, we deduce the integral range $S_{0} \approx$ $3.5210^{-2} \mu \mathrm{m}^{2}$. At the scale of the SEM images $\left(S=153 \mu \mathrm{m}^{2}\right)$ the absolute error in Eq. (5) is $\epsilon_{\mathrm{abs}} \approx 1.1 \%$ so that $f_{0} \approx 9.4 \pm 1.1 \%$.

\section{Conclusion}

The spatial dispersion of hematite nanoparticles in a epoxy resin is accurately modeled by a two-scales microstructure made of spherical "exclusion zones" containing few hematite particles. Use of the granulometry of the embedding (epoxy) phase as criterion is crucial to describe the spatial dispersion of particles. The model's parameters are efficiently optimized using either standard gradient method or the LevenbergMarquardt method.

Parametrized microstructure models are a first step towards designing materials with improved physical properties. 3D microstructure models are conveniently coupled with Fourier numerical methods [18] for solving PDEs on images of complex materials, without meshing. These tools predict the electric [18], elastic [9] or optical [8] responses of composites. Applied to the present work, these methods should allow one to determine how the peculiar multiscale dispersion of nanoparticles affects the material's overall properties. In previous numerical investigations [9], multiscale dispersions have been shown to strongly influence the physical properties of inclusion-matrix coatings.

Acknowledgement This study was made with the support of A.N.R. (Agence Nationale de la Recherche) under grant 20284 (LIMA project). 


\section{REFERENCES}

[1] M. Spuch-Calvar, J. Pérez-Juste, L. M. Liz-Marzán, Hematite spindles with optical functionalities: Growth of gold nanoshells and assembly of gold nanorods, J. of Colloid and Interface Science 310, pp. 297-301 (2007).

[2] Y. Xu, S. Yang, G. Zhang, Y. Sun, D. Gao, Y. Sun, Uniform hematite $\alpha-\mathrm{Fe}_{2} \mathrm{O}_{3}$ nanoparticles: Morphology, size-controlled hydrothermal synthesis and formation mechanism, Materials Letters 65 pp. 1911-1914 (2011).

[3] M. Tadic, N. Citakovic, M. Panjan, B. Stanojevic, D. Markovic, Đ. de Jovanovic, V. Spasojevic, Synthesis, morphology and microstructure of pomegranate-like hematite $\left(\alpha-\mathrm{Fe}_{2} \mathrm{O}_{3}\right)$ superstructure with high coercivity, J. of Alloys and Compounds 543 (5), pp. 118-124 (2012).

[4] G.-S. Park, D. Shindo, Y. Waseda, T. Sugimoto, Internal Structure Analysis of Monodispersed Pseudocubic Hematite Particles by Electron Microscopy, J. of Colloid and interface Science 177, pp. 198-207 (1996).

[5] S. Manickavasagam, C. Saltiel, H. Giesche, Characterization of colloidal hematite particle shape and dispersion behavior, J. of Colloid and Interface Science 280, pp. 417-430 (2004).

[6] X. Huang, J. Guan, Z. Xiao, G. Tong, F. Mou, X. Fan, Flower-like porous hematite nanoarchitectures achieved by complexation-mediated oxidation-hydrolysis reaction, J. of Colloid and Interface Science 357, pp. 36-45 (2007).

[7] A. Jean, D. Jeulin, S. Forest, S. Cantournet, F. N'Guyen, A multiscale microstructure model of carbon black distribution in rubber, J. of Microscopy 241 (3), pp. 243-260 (2011).

[8] D. F. Azzimonti, F. Willot, D. Jeulin, Optical properties of deposit models for paints: fullfields FFT computations and representative volume element, J. of Modern Optics 60 (7), pp. 1-10 (2013).

[9] F. Willot, D. Jeulin, Elastic and electrical behavior of some random multiscale highly- contrasted composites, Int. J. for Multiscale Computational Engineering 9 (3), pp. 305326 (2011).

[10] D. Jeulin, Random structures in physics, in M. Bilodeau, F. Meyer and M.Schmitt, eds., Space structure and Randomness 183, Lecture Notes in Statistics, pp. 183-219, Springer New-York (2005).

[11] M. Ben Achour, A. Chesnaud, A. Thorel, J.-F. Hochepied, L. Lajaunie, F. Willot, E. Couka, A. Paljic, Dielectric properties of hematite nanoparticles with various morphologies, unpublished.

[12] N. Otsu, A threshold selection method from gray-level histograms, IEEE Trans. Sys. Man. Cyber. 9, pp. 62-66 (1979).

[13] F. Willot, L. Gillibert, D. Jeulin, Microstructure-induced hotspots in the thermal and elastic responses of granular media, Int. J. of Solids and Structures 50 (10), pp. 1699-1709 (2013).

[14] W. H. Press, B. P. Flannery, S. A. Teukolsky, W. T. Vetterling, Numerical Recipes Third Edition: The art of scientific computing, Cambridge Universtity Press (2007).

[15] G. Matheron, The Theory of Regionalized Variables and its Applications, Paris School of Mines Publications (1971).

[16] T. Kanit, S. Forest, I. Galliet, V. Mounoury, D. Jeulin, Determination of the size of the representative volume element for random composites: statistical and numerical approach. Int. J. of Solids and Structures 40 (13), pp. 3647-3679 (2003).

[17] T. Kanit, F. N'Guyen, S. Forest, D. Jeulin, M. Reed, S. Singleton, Apparent and effective physical properties of heterogeneous materials: representativity of samples of two materials from food industry. Computer Methods in Applied Mechanics and Engineering (33) 195, pp. 3960-3982 (2006).

[18] F. Willot, B. Abdallah, Y.-P. Pellegrini, Fourier-based schemes with modified Green operator for computing the electrical response of heterogeneous media with accurate local fields. to appear in Int. J. for Numerical Methods in Engineering (doi 10.1002/nme.4641). ArXiv preprint: 1307.1015 . 